\title{
Establishment and validation of a logistic regression model for prediction of septic shock severity in children
}

\author{
Yujie Han, Lili Kang, Xianghong Liu, Yuanhua Zhuang, Xiao Chen and Xiaoying Li*
}

\begin{abstract}
Background: Septic shock is the most severe complication of sepsis, and is a major cause of childhood mortality, constituting a heavy public health burden.

Methods: We analyzed the gene expression profiles of septic shock and control samples from the Gene Expression Omnibus (GEO). Four differentially expressed genes (DEGs) from survivor and control groups, non-survivor and control groups, and survivor and non-survivor groups were selected. We used data about these genes to establish a logistic regression model for predicting the survival of septic shock patients.

Results: Leave-one-out cross validation and receiver operating characteristic (ROC) analysis indicated that this model had good accuracy. Differential expression and Gene Set Enrichment Analysis (GSEA) between septic shock patients stratified by prediction score indicated that the systemic lupus erythematosus pathway was activated, while the limonene and pinene degradation pathways were inactivated in the high score group.

Conclusions: Our study provides a novel approach for the prediction of the severity of pathology in septic shock patients, which are significant for personalized treatment as well as prognostic assessment.

Keywords: Septic shock, Logistic regression model, Survival, Systemic lupus erythematosus pathway, Limonene and pinene degradation pathway
\end{abstract}

\section{Introduction}

Sepsis is an acute organ dysfunction that is secondary to infection [1]. It is a major cause of death in patients with complex conditions including humoral and cellular reactions, inflammatory and anti-inflammatory issues, and circulatory problems [2-4]. The most severe complication of sepsis, septic shock, has a mortality of $20-35 \%$ [5]. Septic shock is characterized by hemodynamic alterations including hypovolemia, decrease in vascular tone, and myocardial depression associated with organ dysfunction [6]. Therapies consist of inhibition of bacterial

*Correspondence: lixiaoyingsd2020@163.com

Department of Neonatal, Qilu Children's Hospital of Shandong University, No. 23976, Huaiyin District, Jinan City 250022, Shandong, People's

Republic of China antioxidant mechanism and biofilm formation, antimicrobials, hyperbaric oxygen and ozone therapies, bacteriophage therapy, etc. [7], and the treatment depends upon the extent of disease progression.

The exploration of prognostic factors for septic shock will be valuable for the assessment of patients and decisions about interventions and treatment adjustment [8]. Several prognostic factors for sepsis and septic shock have been investigated. It has been previously reported that the short-term prognosis of septic shock is influenced by multiorgan failure, concordance of empiric antibiotic treatment with sensitivity testing in vitro, presence of more than two comorbidities, and Karnofsky score, while higher organ failure score, relapse of hematologic disease, Karnofsky score and resistance to treatment are important prognostic factors for long-term prognosis [9]. original author(s) and the source, provide a link to the Creative Commons licence, and indicate if changes were made. The images or other third party material in this article are included in the article's Creative Commons licence, unless indicated otherwise in a credit line to the material. If material is not included in the article's Creative Commons licence and your intended use is not permitted by statutory regulation or exceeds the permitted use, you will need to obtain permission directly from the copyright holder. To view a copy of this licence, visit http://creativecommons.org/licenses/by/4.0/. The Creative Commons Public Domain Dedication waiver (http://creativeco mmons.org/publicdomain/zero/1.0/) applies to the data made available in this article, unless otherwise stated in a credit line to the data. 
Procalcitonin (PCT) has been used as an early diagnostic marker for sepsis [10]. However, individual determination of PCT produces variable results, and may not be an effective diagnostic approach $[11,12]$. Serial determinations of PCT have been shown to independently predict the mortality of severe sepsis [13], but this analysis is complex and time consuming. Circulating histones have been put forward as potential markers for sepsis evolution, but no consistent conclusions have been drawn on the specific concentration of each histone for sepsis monitor [14]. Yan HP et al. suggested that plasma mtDNA might be candidate biomarker for the prognosis of sepsis, but this study lacked serial measurement of mtDNA levels [15].

As the biological processes involved in septic shock are complex, it is proposed that the use of multibiomarkers for the stratification of septic shock may meet both research and clinical needs. In a previous study, 15 candidate biomarkers-CCL3, CCL4, ELA2, FGL2, GZMB, HSPA1B, IL1A, IL8, LCN2, LTF, MMP8, ORM1, RETN, SULF2, and THBS-were selected using a genome-wide expression database [16, 17]. Through integrative bioinformatic methods, Rosier $\mathrm{F}$ et al. screened out some genes related to the death of septic shock patients, and demonstrated that the genetic variation in $\mathrm{CISH}$ resulted in elevated death risk of patients with sepsis [5]. A total of six genes, including CREBBP, WDR82, NCOA1, ASH1L, $T P R$, and $S F 1$, were identified as prognosis-related genes in patients with septic shock via comprehensively analyzing the gene expression spectrum [18]. OLFM4 polymorphisms were found to be able to anticipate the clinical outcome of septic shock patients after major surgery [19]. In our study, a logistic regression model was established using data from four vital genes: GOS2, CTSD, PRUNE2, and $S L C 22 A 4$, to predict the severity of septic shock in children, using the analysis of differentially expressed genes (DEGs) among the survivors, non-survivors and controls. The model presents encouraging predictive value in evaluating severity and lethality of children septic shock, which should be helpful for the disease assessment and treatment management.

\section{Materials and methods}

\section{Study population}

The gene expression profiles of 130 whole blood samples were obtained from Gene Expression Omnibus (GEO) (https://www.ncbi.nlm.nih.gov/geo/, Accession number: GSE26440), including 98 children with septic shock and 32 controls with age from 0 to 10.9 and median age of 2 . The 98 septic shock patients consisted of 81 survivors and 17 non-survivors. Microarray hybridization was carried out using the Human Genome U133 Plus 2.0 GeneChip (Affymetrix, Santa Clara, CA, USA).
The dataset GSE26440 consisted of the grouping information of groups A, B, and C, which could be used directly. Compared with groups $\mathrm{B}$ and $\mathrm{C}$, the patients in group A were younger, with higher disease severity and mortality rate.

\section{Differential expression analysis}

Background correction and normalization of the data were carried out with Affy Bioconductor package. The differentially expressed genes (DEGs) were analyzed using limma $\mathrm{R}$ package. $P$-value $<0.05$ and $|\log 2 \mathrm{FC}|>1$ (FC: Fold Change) were set as thresholds for significantly differential expression.

\section{Candidate genes selection and logistic regression analysis}

The DEGs between survivor and control groups, nonsurvivor and control groups, survivor and non-survivor groups were analyzed to obtain their intersection. Pearson correlation was conducted to calculate the correlation between two genes, and genes with Pearson correlation coefficient larger than 0.8 were excluded.

The logistic regression model was established to predict survival probability using stepwise regression method, with the selected genes as independent variables, and survival/death as dependent variables. Leave-one-out cross validation was performed to evaluate the accuracy of the model. Receiver operating characteristic (ROC) curve was used to assess the discrimination of the model and the optimal cutoff point was obtained.

\section{Function enrichment analysis}

The blood samples from patients with septic shock were evaluated by logistic regression model to obtain the prediction scores. According to the prediction scores, septic shock samples were divided into high score and low score groups. Gene Set Enrichment Analysis (GSEA) of the DEGs between two groups was carried out to select the significantly enriched Kyoto Encyclopedia of Genes and Genomes (KEGG) pathways.

\section{Results}

DEGs between survivor and control, non-survivor and control, survivor and non-survivor groups

We analyzed the DEGs between survivor and control, non-survivor and control, survivor and non-survivor groups, and obtained 575 (Fig. 1A), 651 (Fig. 1B), 75 (Fig. 1C) DEGs respectively. The 11 common DEGs of the three groups were as follows: PRUNE2, LCN2, HSPA1A, SLC22A4, C1QC, CTSD, CEP55, RAB13, ORM1, GOS2, and CLEC5A (Fig. 2). 


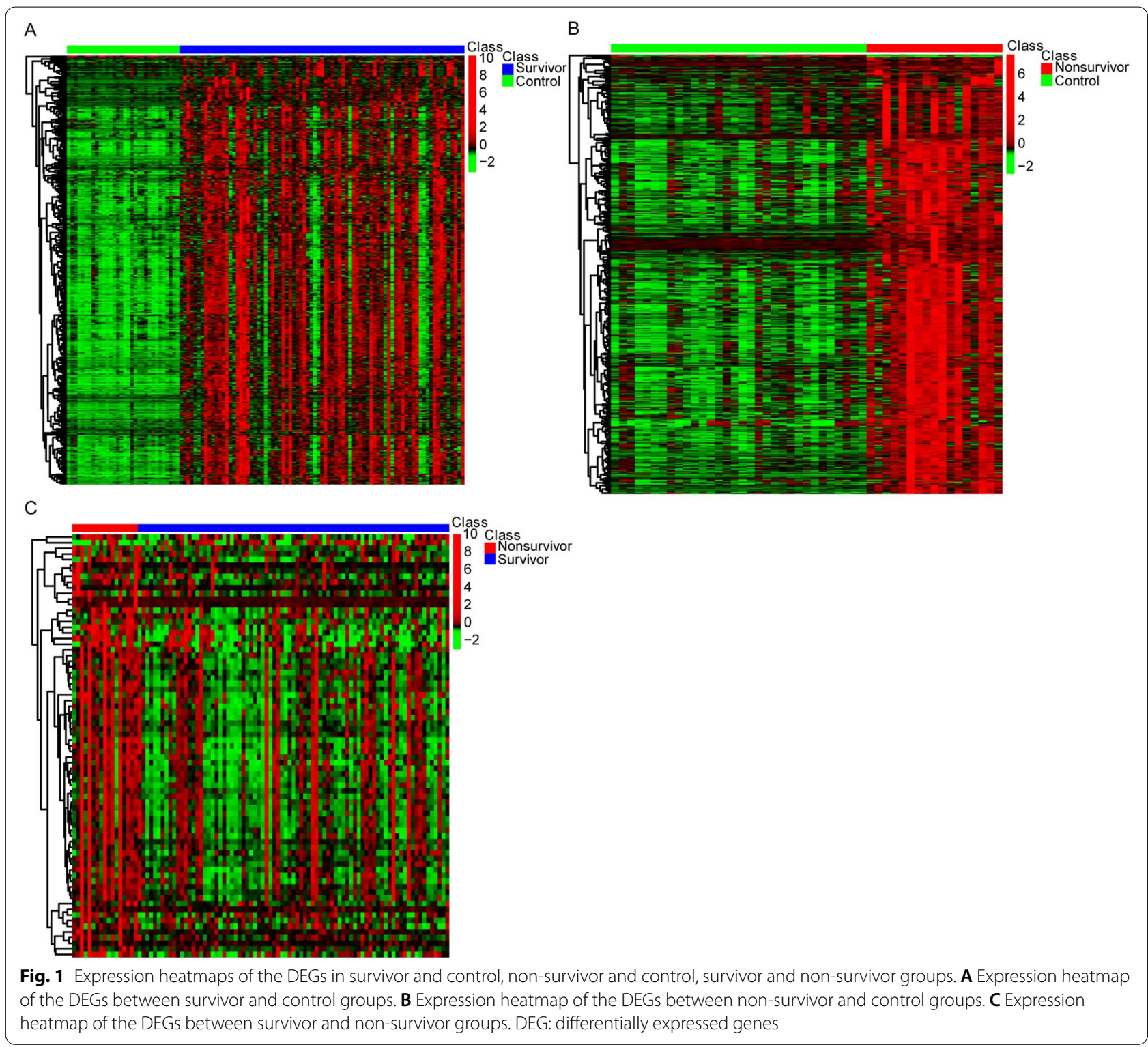

\section{Establishment of logistic regression model}

Correlation analysis of the 11 genes indicated the Pearson correlation coefficients of them were all less than 0.8 (Fig. 3A), which could be used for logistic regression model. Four genes G0S2 $(p=0.00281)$, CTSD $(p=0.01326), \quad$ PRUNE2 $\quad(p=0.03506), \quad$ SLC22A4 $(p=0.00615)$ were selected after stepwise logistic regression analysis for the construction of the predictive model. All samples in the dataset were assigned a risk score which represents death risk through the predictive model based on their expression levels of the four genes. Leave-one-out analysis of the risk score cross validation showed the area under ROC curve (AUC) was 0.873 and the optimal cutoff value of ROC was $0.188(0.840,0.824)$
(Fig. 3B). In addition, samples in the original study of GSE26440 dataset [20] were classified into three subgroups which named group A, group B, and group C through unsupervised hierarchical clustering based on the empiric, discovery-oriented gene expression. Here, the independence of prediction score, age, and grouping for survival/death prediction was investigated by logistic regression analysis with prediction score, age, and grouping as independent variables and survival/death as responsive variables. Figure $3 \mathrm{C}$ suggested age was not a risk factor for survival (OR: 0.93, 95\% CI: 0.67-1.22); for the subclasses classified in previous study, the mortality of patients in group $\mathrm{C}$ was significantly lower than that in group A (OR: 0.042, 95\% CI: 0.0013-0.5) and no 

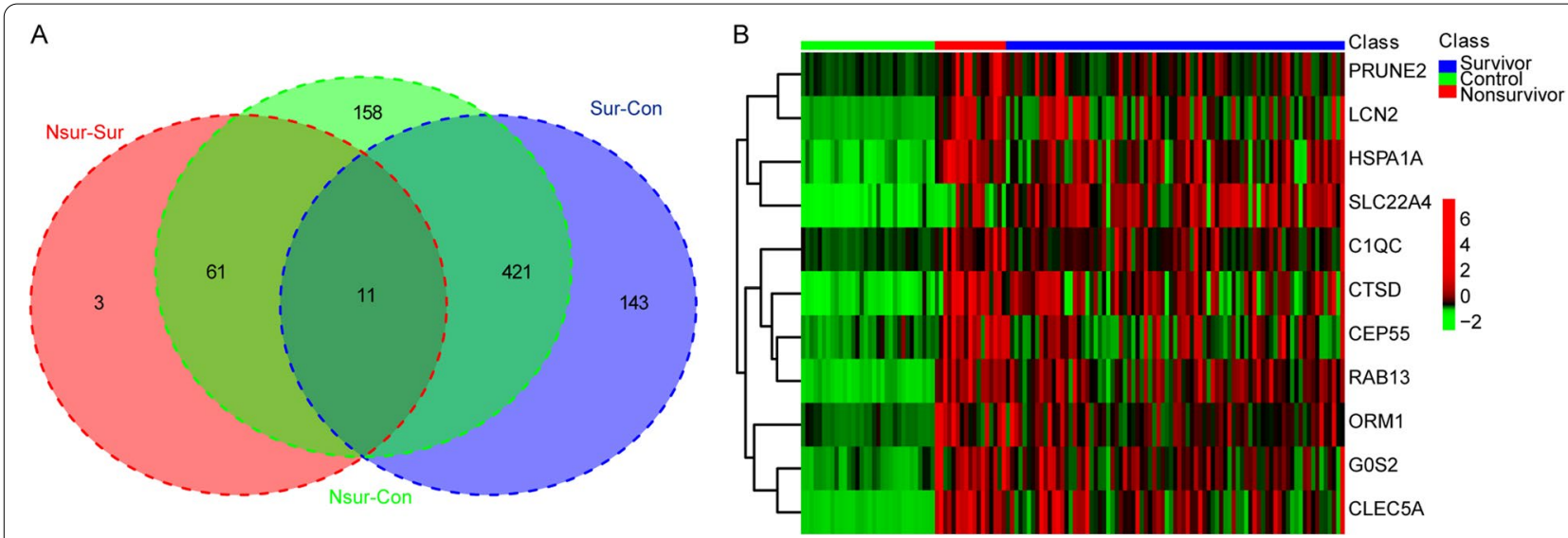

Fig. 2 Common DEGs in survivor and control, non-survivor and control, survivor and non-survivor groups. A There were 575 DEGs between survivor and control groups, 651 DEGs between non-survivor and control groups, 75 DEGs between survivor and non-survivor groups, and 11 common DEGs among the three groups. B Expression heatmap of the 11 common DEGs (PRUNE2, LCN2, HSPA1A, SLC22A4, C1QC, CTSD, CEP55, RAB13, ORM1, GOS2, and CLEC5A). DEG: differentially expressed genes

significant difference in survival was observed between groups B and A (OR: 0.44, 95\% CI: 0.065-2.73) [20]. The prediction score was proved to be an accurate indicator for survival (OR: 3179, 95\% CI: 126-310000).

Systemic lupus erythematosus pathway was up-regulated while limonene and pinene degradation pathway was down-regulated in high prediction score group

The septic shock samples were divided into high score and low score groups based on the prediction scores, with mean value of the prediction scores as threshold. There were 69 DEGs (Table S1) between high score and low score groups, with the expression heatmap shown in Fig. 4A. GSEA revealed 5 signal pathways were significantly up-regulated in high prediction score group, including systemic lupus erythematosus, cell cycle, complement and coagulation cascades, DNA replication, renin angiotensin system (RAS) pathways and the upregulation of systemic lupus erythematosus pathway was the most obvious (Fig. 4B, C), while the limonene and pinene degradation pathway was significantly down-regulated (Fig. 4D).

\section{Discussion}

Septic shock is a significant cause of childhood morbidity and mortality all over the world, and generates a heavy public health burden $[21,22]$. Septic shock remains the fifth most important cause of years of productive life lost by premature mortality [22]. Septic shock syndrome has multiple causes, and is a major inducer of clinical failure involving the inflammatory or immune systems [23]. A single therapy that targets the inflammatory or immune system may not be valid for a heterogeneous population of patients with septic shock. A classification of septic shock patients will therefore be beneficial for the development of personalized treatments, as well as for prognostic assessment [20].

In our study, the analysis of DEGs between survivor and control groups, non-survivor and control groups, and survivor and non-survivor groups was performed, and 11 common genes were identified. After logistic regression analysis, four genes-GOS2, CTSD, PRUNE2, and SLC22A4-were selected with which to establish a model for predicting the severity of septic shock in patients. Leave-one-out cross validation and ROC analysis indicated that the logistic regression model had high accuracy in predicting the survival of septic shock patients. Additionally, samples for gene expression profiling are blood from septic shock patients, which would make the model possible to serve as a noninvasive means for survival evaluation.

Among the four genes, CTSD has been proved to be associated with septic shock. It has been shown that TGase 2 could protect liver from the septic shock induced by TNF- $\alpha$ through decreasing CTSD expression level [24]. Moreover, compared with untreated septic shock cats, the septic shock cats treated by anisodamine, an alkaloid used for septic shock treatment, had decreased cathepsin D (encoded by CTSD) activity [25]. Although there are no direct evidences on the relationship between G0S2, PRUNE2, SLC22A4 and septic shock or sepsis, several researches indicated their indirect association. PRUNE2 is considered as a regulator of Rho signaling [26]. It is known that Rho could interact with Rho kinase, which has the therapeutic effects on alleviations of inflammation and coagulation dysfunction in sepsis 


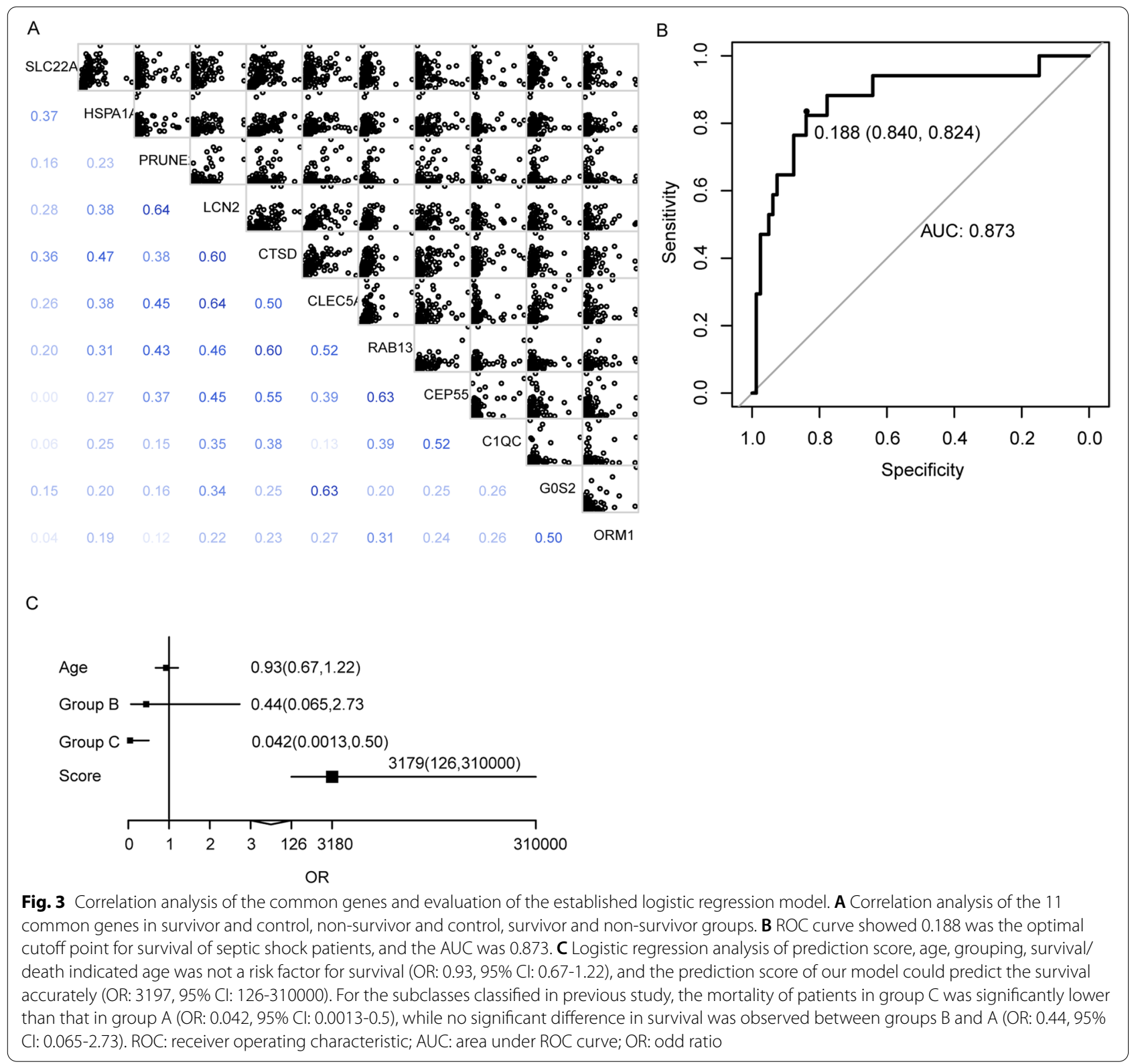

and is recognized as the promising therapeutic target [27, 28]. Hence, PRUNE2 might participate in sepsis through regulating Rho signaling and Rho kinase. $S L C 22 A 4$, also known as OCTN1, is regulated by several factors including inflammatory cytokines, and related to inflammatory diseases [29]. It is suggested that inflammatory cytokines play a pivotal role in the organ damage during inflammatory disease of septic shock [30]. Thus, we inferred that SLC22A4 might be involved in septic shock with the interaction of inflammatory cytokines. A previous study showed that overexpression of $\mathrm{Bcl}-2$ in septic mice could improve the mortality with anti-apoptosis effects on the intestinal epithelial cell [31]. As we know, GOS2 inhibits the anti-apoptosis function of $B c l-2$ by suppressing the formation of $\mathrm{Bcl}-2 / \mathrm{Bax}$ heterodimeric complexes [32]. These results indicated that GOS2 might play a pro-apoptosis role in the intestinal epithelium of sepsis, contributing to a high mortality. Taken together, the up-regulation of these four genes may play a role in immunosuppression of sepsis by interfering with downstream signal pathways or macromolecular complexes formation.

The development of a system of classification of septic shock patients could increase the efficiency of hemodynamic management [33], and numerous efforts have been made to search for biomarkers for septic shock classification. Biomarkers identified to date include IL- 8 and 

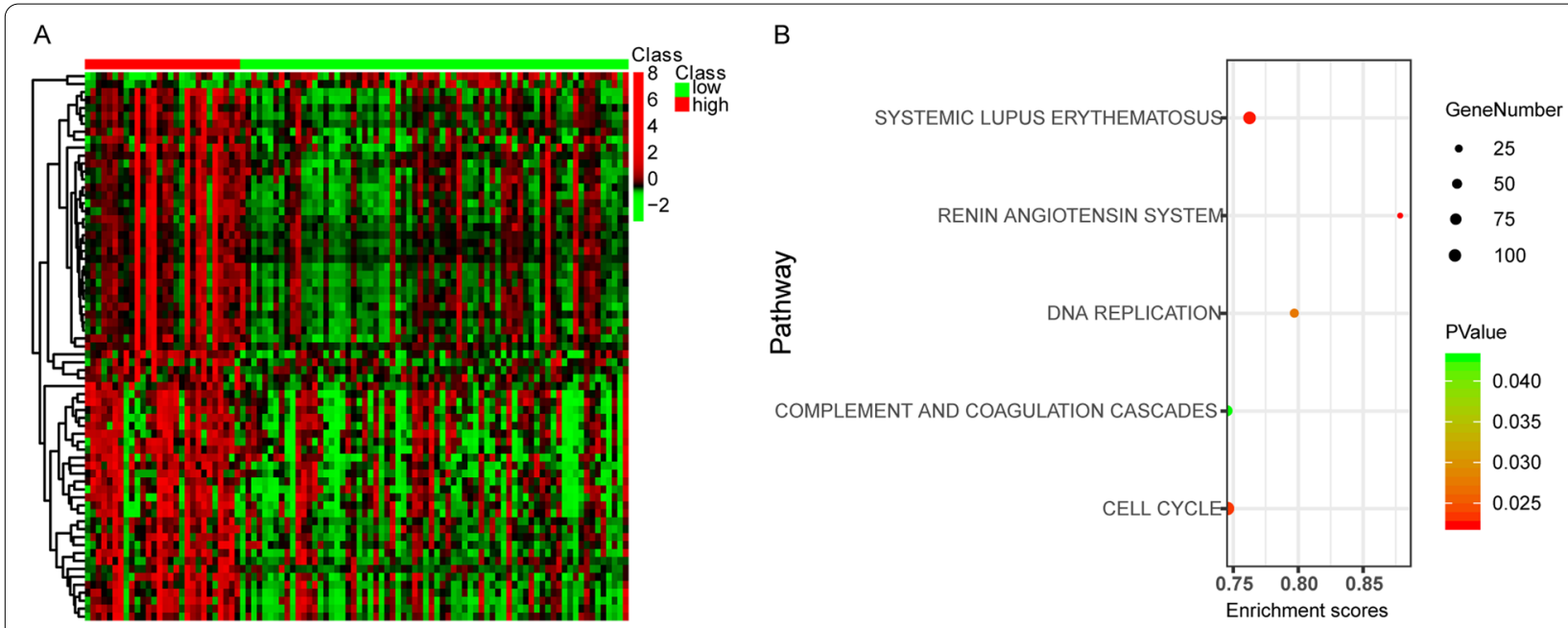

C

D
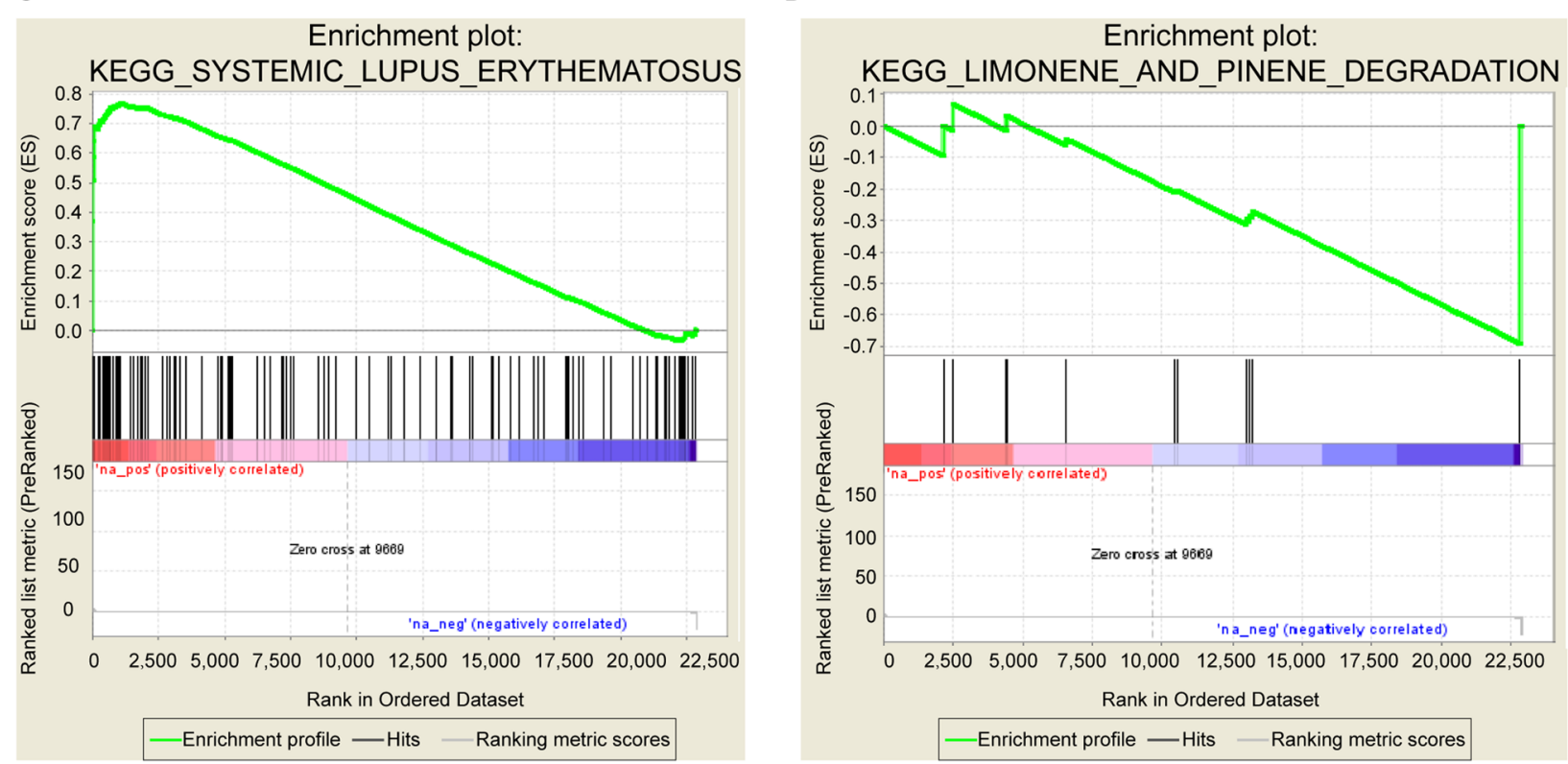

Fig. 4 Systemic lupus erythematosus pathway was significantly up-regulated while limonene and pinene degradation pathway was significantly down-regulated in high-prediction score group. A Expression heatmap of the 69 DEGs between high score and low score groups. B Up-regulation of 5 signal pathways were observed in high prediction score group. C Systemic lupus erythematosus pathway was significantly up-regulated in high prediction score group. D Limonene and pinene degradation pathway was significantly down-regulated in high prediction score group. DEG: differentially expressed genes

CCL4 [17]. However, as septic shock is a complex and heterogeneous condition, the classification made using these biomarkers has been shown to be simplistic, with limited sensitivity, specificity, and positive predictive value [34-36]. A potential alternative to this approach is the use of genome-wide expression profiles. The significant expression of genes that are grouped in a score could improve the validity of each individual to predict the survival. Wong HR et al. identified three putative subclasses:
A, B, and C, by analyzing DEGs between septic shock patients and controls. The data collected by Wong HR et al. in infants are well done and very informative in absence of major comorbidities, as their transposition to the adults may be limited by the chronic diseases and chronic treatment, or comorbidities [20]. Patients in subclass A showed more severe illness and higher mortality rates at a younger age than patients in subclasses $B$ and $C$ [20]. In contrast, the model we developed to predict the 
survival of septic shock patients takes into account the difference between survivors and non-survivors.

We predicted the scores of septic shock patients using our model, and divided them into high-scoring and lowscoring groups, based on the prediction scores. A total of 69 DEGs were observed between the two groups, and the DEGs were significantly enriched in six signal pathways. Among them, the pathways such as systemic lupus erythematosus and RAS were significantly up-regulated, while the limonene and pinene degradation pathway was significantly down-regulated.

Systemic lupus erythematosus is a chronic autoimmune disorder characterized by the production of autoantibodies against nuclear and cytoplasmic antigens [37]. Infection caused by common opportunistic agents is the major reason for morbidity and mortality in immunocompromised systemic lupus erythematosus patients [38-40]. Sepsis is one of the leading causes for death of systemic lupus erythematosus patients [41]. A patient suffering from systemic lupus erythematosus with septic shock caused by a virus has been reported [42]. A high incidence of Salmonella infections, which may develop sepsis, has been reported in systemic lupus erythematosus patients [40]. Immunosuppression is considered to be the pivotal host response in sepsis, contributing to the susceptibility to infection of systemic lupus erythematosus patients, and is recognized as one of the vital variables for systemic lupus erythematosus [43-45]. It is hypothesized that the immunosuppression caused by septic shock may lead to the up-regulation of the systemic lupus erythematosus pathway.

As a crucial neuroendocrine system, RAS has been a hotspot in the research area of sepsis. The angiotensin I transforms into angiotensin II when the blood pressure is reduced; Angiotensin II, the main active peptide in RAS, plays a key role in increasing the blood pressure with the combination of ATR [46]. Angiotensin II probably contributes to the aggravation of inflammatory reaction by enhancing the chemokines and proinflammatory cell factors synthesis [47], while sepsis is characterized by severe inflammatory reaction [48]. Tamion $\mathrm{F}$ et al. showed that RAS was implicated in the development of sepsis, and sepsis might be related to elevated RAS expression levels [49]. This may explain why the septic shock patients with high prediction score has significantly up-regulated RAS pathway in our study.

Limonene is a natural component in citrus fruits which exhibits anti-inflammatory function and could alleviate inflammation by targeting $\mathrm{A}_{2 \mathrm{~A}}$ receptors [50]. $\alpha$-pinene is a compound in the oils of coniferous trees as well as a main ingredient of volatiles that are extracted from various types of trees [51]. It has multiple biological functions including the an-inflammatory effect. Kim DS et al. found that $\alpha$-pinene could inhibit the inflammatory response and is a promising alternative for the treatment of inflammation [52]. Considering the anti-inflammatory effects of limonene and pinene, it is assumed that the down-regulated limonene and pinene degradation pathway in the high-scoring group may increase the levels of limonene and pinene to antagonize the inflammatory response caused by septic shock. However, the underlying mechanism still warrants further investigation.

In conclusion, we established a logistic regression model for predicting the survival of pediatric septic shock patients, and validated its accuracy. In septic shock patients stratified by prediction score, the systemic lupus erythematosus pathway was activated, while the limonene and pinene degradation pathway was inactivated in the high-prediction score group. Our research provides a novel approach to the assessment of the survival status of septic shock patients, and can be valuable for the development of personalized treatment.

\section{Supplementary Information}

The online version contains supplementary material available at https://doi. org/10.1186/s41065-021-00206-9.

Additional file 1: Table S1. DEGs between high and low risk score sepsis samples.

\section{Acknowledgements}

Not applicable.

\section{Authors' contributions}

Conceptualization: Yujie Han and Xiaoying Li. Data curation and Formal analysis: Lili Kang and Xianghong Liu. Software and Methodology: Yuanhua Zhuang and Xiao Chen. Writing-original draft: All authors. Writing-review \& editing:

Xiaoying Li. All authors read and approved the final manuscript.

Funding

Not applicable.

Availability of data and materials

All data generated or analyzed during this study are included in this published article.

\section{Declarations}

Ethics approval and consent to participate

Not applicable.

Consent for publication

Not applicable.

Competing interests

The authors declare that they have no competing interests.

Received: 15 June 2021 Accepted: 14 October 2021

Published online: 12 November 2021 


\section{References}

1. Prescott HC, Angus DC. Enhancing recovery from sepsis: a review. JAMA 2018;319(1):62-75. https://doi.org/10.1001/jama.2017.17687.

2. Eisen DP, Leder $K$, Woods RL, et al. Effect of aspirin on deaths associated with sepsis in healthy older people (ANTISEPSIS): a randomised, doubleblind, placebo-controlled primary prevention trial. Lancet Respir Med. 2021;9(2):186-95. https://doi.org/10.1016/S2213-2600(20)30411-2.

3. Drosatos K, Lymperopoulos A, Kennel PJ, et al. Pathophysiology of sepsisrelated cardiac dysfunction: driven by inflammation, energy mismanagement, or both? Curr Heart Fail Rep. 2015;12(2):130-40. https://doi.org/10. 1007/s11897-014-0247-z.

4. Dietrich M, Marx S, von der Forst M, et al. Bedside hyperspectral imaging indicates a microcirculatory sepsis pattern - an observational study. Microvasc Res. 2021;136:104164. https://doi.org/10.1016/j.mvr.2021. 104164.

5. Rosier F, Brisebarre A, Dupuis C, et al. Genetic predisposition to the mortality in septic shock patients: from GWAS to the identification of a regulatory variant modulating the activity of a CISH enhancer. Int J Mol Sci. 2021;22(11). https://doi.org/10.3390/ijms22115852.

6. Backer DD, Cortes DO, Donadello K, Vincent JL. Pathophysiology of microcirculatory dysfunction and the pathogenesis of septic shock. Virulence. 2013;5:73-9. https://doi.org/10.4161/viru.26482.

7. Minasyan H. Sepsis and septic shock: pathogenesis and treatment perspectives. J Crit Care. 2017;40:229-42. https://doi.org/10.1016/j.jcrc.2017. 04.015 .

8. de Azevedo JR, Torres OJ, Beraldi RA, Ribas CA, Malafaia O. Prognostic evaluation of severe sepsis and septic shock: Procalcitonin clearance vs $\Delta$ sequential organ failure assessment. J Crit Care. 2015;30:219.e9-e12. https://doi.org/10.1016/j.jcrc.2014.08.018.

9. Waszczuk-Gajda A, Wiktor JW. Prognostic factors in the survival of patients with blood disorders recovering from septic shock. Hematology. 2017;22:292-8. https://doi.org/10.1080/10245332.2016.1253521.

10. Mahadev N, Raheem SA, Haddannavar VB. A study of serum procalcitonin (PCT) as a diagnostic biomarker in sepsis in ICU. J Evid Based Med Healthc. 2017;1. https://doi.org/10.18410/jebmh/2017/141.

11. Saikant R, Ravindran S, Vijayan A, Maya V, Lakshmi S, Kartik R, et al. Response of letter to the editor on Procalcitonin: a promising diagnostic marker for sepsis and antibiotic therapy. J Intensive Care. 2017;5:68. https://doi.org/10.1186/s40560-017-0260-x.

12. Aydemir C, Aydemir H, Kokturk F, Kulah C, Mungan AG. The cut-off levels of procalcitonin and $\mathrm{C}$-reactive protein and the kinetics of mean platelet volume in preterm neonates with sepsis. BMC Pediatr. 2018;18:253. https://doi.org/10.1186/s12887-018-1236-2.

13. Schuetz $P$, Birkhahn $R$, Sherwin $R$, Jones AE, Singer A, Kline JA, et al. Serial Procalcitonin predicts mortality in severe sepsis patients: results from the multicenter procalcitonin MOnitoring SEpsis (MOSES) study. Crit Care Med. 2017:45:781-9. https://doi.org/10.1097/CCM.0000000000002321.

14. Garcia-Gimenez JL, Roma-Mateo C, Carbonell N, Palacios L, Peiró-Chova L, García-López E, et al. A new mass spectrometry-based method for the quantification of histones in plasma from septic shock patients. Sci Rep. 2017:7:10643. https://doi.org/10.1038/s41598-017-10830-z.

15. Yan HP, Li M, Lu XL, Zhu YM, Ou-Yang WX, Xiao ZH, et al. Use of plasma mitochondrial DNA levels for determining disease severity and prognosis in pediatric sepsis: a case control study. BMC Pediatr. 2018;18:267. https:// doi.org/10.1186/s12887-018-1239-z.

16. Kaplan JM, Wong HR. Biomarker discovery and development in pediatric critical care medicine. Pediatr Crit Care Med. 2011;12:165-73. https://doi. org/10.1097/PCC.0b013e3181e28876.

17. Standage SW, Wong HR. Biomarkers for pediatric sepsis and septic shock. Expert Rev Anti-Infect Ther. 2011;9:71-9. https://doi.org/10.1586/eri.10. 154.

18. Hu Y, Cheng L, Zhong W, et al. Bioinformatics analysis of gene expression profiles for risk prediction in patients with septic shock. Med Sci Monit. 2019:25:9563-71. https://doi.org/10.12659/MSM.918491.

19. Perez-Garcia F, Resino S, Gomez-Sanchez E, et al. OLFM4 polymorphisms predict septic shock survival after major surgery. Eur J Clin Investig. 2021;51(4):e13416. https://doi.org/10.1111/eci.13416.

20. Wong HR, Cvijanovich N, Lin R, Allen GL, Thomas NJ, Willson DF, et al. Identification of pediatric septic shock subclasses based on genomewide expression profiling. BMC Med. 2009;7:34-40. https://doi.org/10 1186/1741-7015-7-34.
21. Zimmerman JJ. Adjunctive steroid therapy for treatment of pediatric septic shock. Pediatr Clin N Am. 2017:64:1133-46. https://doi.org/10.1016/j. pcl.2017.06.010.

22. Seymour CW, Rosengart MR. Septic shock: advances in diagnosis and treatment. J Am Med Assoc. 2015;314:708-17. https://doi.org/10.1001/ jama.2015.7885.

23. Murray CJ, Atkinson C, Bhalla K, Birbeck G, Burstein R, Chou D, et al. The state of US health, 1990-2010: burden of diseases, injuries, and risk factors. J Am Med Assoc. 2013;310:591-608. https://doi.org/10.1001/jama.2018. 0158

24. Yoo H, Ahn ER, Kim SJ, Lee SH, Oh SH, Kim SY. Divergent results induced by different types of septic shock in transglutaminase 2 knockout mice. Amino Acids. 2013;44:189-97. https://doi.org/10.1007/ s00726-012-1412-x.

25. Su J, Hock CE, Lefer AM. Beneficial effect of anisodamine in hemorrhagic shock. Naunyn Schmiedeberg's Arch Pharmacol. 1984;325:360-5. https:// doi.org/10.1007/BF00504382.

26. Zhao LR, Tian W, Wang GW, Chen KX, Yang JL. The prognostic role of PRUNE2 in leiomyosarcoma. Chin J Cancer. 2013;32:648-52. https://doi. org/10.5732/cjc.013.10069.

27. Wang Y, Braun OO, Zhang S, Norstrom E, Thorlacius H. Thrombin generation in abdominal sepsis is rho-kinase-dependent. Biochem Biophys Res Commun. 2015;460:691-6. https://doi.org/10.1016/j.bbrc.2015.03.091.

28. McGown CC, Brown NJ, Hellewell PG, Brookes ZL. ROCK induced inflammation of the microcirculation during endotoxemia mediated by nitric oxide synthase. Microvasc Res. 2011;81:281-8. https://doi.org/10.1016/j. mvr.2011.02.003.

29. Maeda T, Hirayama M, Kobayashi D, Miyazawa K, Tamai I. Mechanism of the regulation of organic cation/carnitine transporter 1 (SLC22A4) by rheumatoid arthritis-associated transcriptional factor RUNX1 and inflammatory cytokines. Drug Metab Dispos. 2007;35:394-401. https://doi.org/ 10.1124/dmd.106.012112

30. Liu J, Wang J, Luo H, Li Z, Zhong T, Tang J, et al. Screening cytokine/ chemokine profiles in serum and organs from an endotoxic shock mouse model by LiquiChip. Sci China Life Sci. 2017;60:1242-50. https://doi.org/ 10.1007/s11427-016-9016-6.

31. Coopersmith CM, Chang KC, Swanson PE, Tinsley KW, Stromberg PE, Buchman TG, et al. Overexpression of $\mathrm{BCl}-2$ in the intestinal epithelium improves survival in septic mice. Crit Care Med. 2002;30:195-201. https:// doi.org/10.1097/00003246-200201000-00028.

32. Welch C, Santra MK, El-Assaad W, Zhu X, Huber WE, Keys RA, et al. Identification of a protein, GOS2, that lacks BCl-2 homology domains and interacts with and antagonizes Bcl-2. Cancer Res. 2009;69:6782-9. https:// doi.org/10.1158/0008-5472.CAN-09-0128.

33. Jellema WT, Groeneveld ABJ, Wesseling KH, Thijs LG, Westerhof $N$, van Lieshout JJ. Heterogeneity and prediction of hemodynamic responses to dobutamine in patients with septic shock. Crit Care Med. 2006:34:2392-8. https://doi.org/10.1097/01.CCM.0000233871.52553.CD.

34. Marshall JC, Vincent JL, Fink MP, Cook DJ, Rubenfeld G, Foster D, et al. Measures, markers, and mediators: toward a staging system for clinical sepsis. A report of the fifth Toronto sepsis roundtable, Toronto, Ontario, Canada, October 25-26, 2000. Crit Care Med. 2003;31:1560-7. https://doi. org/10.1097/01.ccm.0000065186.67848.3a.

35. Wong HR, Cvijanovich N, Wheeler DS, Bigham MT, Monaco M, Odoms K, et al. Interleukin-8 as a stratification tool for interventional trials involving pediatric septic shock. Am J Respir Crit Care Med. 2008;178:276-82. https://doi.org/10.1164/rccm.200801-1310C.

36. Nowak JE, Wheeler DS, Harmon KK, Wong HR. Admission chemokine (C-C motif) ligand 4 levels predict survival in pediatric septic shock. Pediatr Crit Care Med. 2010;11:213-6. https://doi.org/10.1097/PCC.0b013e3181 b8076c.

37. Fortuna G, Brennan MT. Systemic lupus erythematosus: epidemiology, pathophysiology, manifestations, and management. Dent Clin N Am. 2013:57:631-55. https://doi.org/10.1016/j.cden.2013.06.003.

38. Naveau C, Houssiau F. Pneumococcal sepsis in patients with systemic lupus erythematosus. Lupus. 2005;14:903-6. https://doi.org/10.1191/ 0961203305lu2242xx

39. Holvast A. Safety and efficacy of influenza vaccination in systemic lupus erythematosus patients with quiescent disease. Ann Rheum Dis. 2005:65:913-8. https://doi.org/10.1136/ard.2005.043943. 
40. Green L, Vinker S. Recurrent salmonella sepsis with different species in a systemic lupus erythematosus patient. Clin Rheumatol. 1996;15:72-4. https://doi.org/10.1007/BF02231690.

41. Shih-Chao K, Shinn-Jang H, Yu-Sheng C, Chou CT, Tsai CY. Characteristics of comorbidities and costs among patients who died from systemic lupus erythematosus in Taiwan. Arch Med Sci. 2012;4:690-6. https://doi. org/10.5114/aoms.2012.30293.

42. Tselios K, Tsioka R, Sarantopoulos A, Mouloudi E, Boura P. Influenza A/ H1N1 septic shock in a patient with systemic lupus erythematosus. A case report. BMC Infect Dis. 2011;11:358. https://doi.org/10.1186/ 1471-2334-11-358.

43. Boomer JS, To K, Chang KC, Takasu O, Osborne DF, Walton AH, et al. Immunosuppression in patients who die of sepsis and multiple organ failure. J Am Med Assoc. 2011;306:2594. https://doi.org/10.1001/jama. 2011.1829.

44. Hotchkiss R. Sepsis-induced apoptosis causes progressive profound depletion of B and CD4+ T lymphocytes in humans. J Immunol. 2001;166:6952-63. https://doi.org/10.4049/jimmunol.166.11.6952.

45. Orit B, Ella A, Jacob D, Victor N, Mahmoud AS. Mortality due to sepsis in patients with systemic lupus erythematosus and rheumatoid arthritis. Israel Med Assoc J. 2014;16:634-5.

46. Zhang W, Chen X, Huang $L$, et al. Severe sepsis: low expression of the renin-angiotensin system is associated with poor prognosis. Exp Ther Med. 2014;7(5):1342-8. https://doi.org/10.3892/etm.2014.1566.

47. Suzuki Y, Ruiz-Ortega M, Lorenzo O, et al. Inflammation and angiotensin II. Int J Biochem Cell Biol. 2003;35(6):881-900. https://doi.org/10.1016/ s1357-2725(02)00271-6.
48. Aliomrani M, Sepand MR, Mirzaei HR, et al. Effects of phloretin on oxidative and inflammatory reaction in rat model of cecal ligation and puncture induced sepsis. Daru. 2016;24(1):15. https://doi.org/10.1186/ s40199-016-0154-9.

49. Tamion F, Le Cam-Duchez V, Menard JF, et al. Erythropoietin and renin as biological markers in critically ill patients. Crit Care. 2004;8(5):R328-35. https://doi.org/10.1186/cc2902.

50. Patel $M$, Narke D, Kurade $M$, et al. Limonene-induced activation of A2A adenosine receptors reduces airway inflammation and reactivity in a mouse model of asthma. Purinergic Signal. 2020;16(3):415-26. https:// doi.org/10.1007/s11302-020-09697-z.

51. Bouzenna H, Hfaiedh N, Giroux-Metges MA, et al. Potential protective effects of alpha-pinene against cytotoxicity caused by aspirin in the IEC-6 cells. Biomed Pharmacother. 2017;93:961-8. https://doi.org/10.1016/j. biopha.2017.06.031.

52. Kim DS, Lee HJ, Jeon YD, et al. Alpha-pinene exhibits anti-inflammatory activity through the suppression of MAPKs and the NF-kappaB pathway in mouse peritoneal macrophages. Am J Chin Med. 2015;43(4):731-42. https://doi.org/10.1142/S0192415X15500457.

\section{Publisher's Note}

Springer Nature remains neutral with regard to jurisdictional claims in published maps and institutional affiliations.
Ready to submit your research? Choose BMC and benefit from:

- fast, convenient online submission

- thorough peer review by experienced researchers in your field

- rapid publication on acceptance

- support for research data, including large and complex data types

- gold Open Access which fosters wider collaboration and increased citations

- maximum visibility for your research: over 100M website views per year

At BMC, research is always in progress.

Learn more biomedcentral.com/submissions 